\title{
Nonlocal Problem for Complete Second-Order Hyperbolic Operator-Differential Equations with Variable Domains
}

\author{
N. A. Khatimtsov and F. E. Lomovtsev \\ Belarus State University, Minsk, Belarus
}

Received April 2, 2009

\begin{abstract}
We prove the well-posed solvability (in the strong sense) of complete second-order hyperbolic operator-differential equations with variable domains of unbounded operator coefficients under nonlocal initial conditions. We are the first to establish the well-posed solvability of the mixed problem for the complete string vibration equation with nonstationary boundary conditions and nonlocal initial conditions.
\end{abstract}

DOI: $10.1134 /$ S0012266111040069

\section{INTRODUCTION}

An abstract Love equation with an unbounded constant operator coefficient under nonlocal initial conditions was studied in [1]. Second-order hyperbolic operator-differential equations with variable domains of unbounded operator coefficients were investigated in $[2,3]$ only under local initial conditions.

The aim of the present paper is to develop a method for studying strong well-posedness of hyperbolic differential equations with variable domains of unbounded operator coefficients in the case of nonlocal initial conditions. Our method generalizes and develops the well-known energy inequality method [1-3]. In the present paper, we prove the existence, uniqueness, and continuous dependence of strong solutions of complete second-order hyperbolic operator-differential equations with variable domains of unbounded operator coefficients under nonlocal initial conditions. The uniqueness and continuous dependence of their strong solutions follows from the energy inequality proved below. Unbounded operators with variable domains are not differentiable with respect to time in the conventional sense, and hence the present paper, unlike [1], makes use of abstract smoothing operators [2] to derive the a priori estimate. By virtue of the energy inequality, to prove that the problem is solvable everywhere, it suffices to prove that the range of the operator of the problem is dense. The proof of the latter claim in [1] on the basis of integral averaging operators and a dualtype inequality cannot be used if the operators have variable domains. Therefore, we have found new techniques for proving this with the use of the new Lemma 6 on the adjoint operator. By using the obtained abstract theorems, we prove the well-posed solvability of an earlier-unstudied mixed problem for the complete string vibration equation with time-dependent boundary conditions and nonlocal initial conditions.

\section{STATEMENT OF THE PROBLEM}

On a bounded interval $] 0, T[$, consider the complete hyperbolic operator-differential equations

$$
\left.\mathcal{L}(t) u \equiv \frac{d^{2} u(t)}{d t^{2}}+A_{1}(t) \frac{d u(t)}{d t}+A(t) u(t)+B(t) \frac{d u(t)}{d t}+B_{0}(t) u(t)=f(t), \quad t \in\right] 0, T[,
$$

with the time-nonlocal conditions

$$
l_{0} u \equiv u(0)-\mu u(T)=\varphi, \quad l_{1} u \equiv \frac{d u(0)}{d t}-\mu \frac{d u(T)}{d t}=\psi, \quad|\mu|<1 .
$$


Here $u$ and $f$ are abstract functions of $t$ ranging in some Hilbert space $H$ with inner product $(\cdot, \cdot)$ and norm $|\cdot|$. For each $t$, the coefficients $A_{1}(t), A(t)$, and $B_{0}(t)$ are linear unbounded operators in $H$ with $t$-dependent domains $D\left(A_{1}(t)\right), D(A(t))$, and $D\left(B_{0}(t)\right)$, respectively, and $\mu$ is a complex parameter. Let the following conditions be satisfied.

$A_{1}$. For each $t \in[0, T]$, the operators $A(t)$ on $D(A(t))$ are self-adjoint and positive in the Hilbert space $H$.

$A_{2}$. In the space $H$, for all $t \in[0, T]$, there exist bounded inverse operators

$$
A^{-1}(t) \in B([0, T], \mathcal{L}(H)),
$$

which are strongly continuous with respect to $t$ and have bounded strong $t$-derivative [4]

$$
d A^{-1}(t) / d t \in B([0, T], \mathcal{L}(H))
$$

satisfying the inequality

$$
-\left(\left(d A^{-1}(t) / d t\right) g, g\right) \leq c_{1}\left(A^{-1}(t) g, g\right) \quad \forall g \in H .
$$

$A_{3}$. For all $t \in[0, T]$, the domains satisfy the inclusion $D(A(t)) \subset D\left(A_{1}(t)\right)$; moreover,

$$
A_{1}(t) A^{-1 / 2}(t) \in B([0, T], \mathcal{L}(H)),
$$

where $A^{-1 / 2}(t)$ is the inverse of the square root $A^{1 / 2}(t)$ of $A(t)$, and

$$
-\operatorname{Re}\left(A_{1}(t) u, u\right) \leq c_{2}|u|^{2} \quad \forall u \in D(A(t)) .
$$

$A_{4}$. There exists a Banach space $V$ and a $t$-independent linear bounded operator $\tilde{A}$ from $V$ to $H$ such that the inclusion $D(A(t)) \subset V \subset H$ holds for each $t \in[0, T]$ and the operators $A(t)$ are the restrictions of the operator $\tilde{A}$ to $D(A(t))$; i.e., $\tilde{A} u=A(t) u$ for all $u \in D(A(t))$.

$A_{5}$. For almost all $\left.t \in\right] 0, T\left[\right.$, the operators $d A^{-1}(t) / d t$ in $H$ have bounded strong $t$-derivative $d^{2} A^{-1}(t) / d t^{2} \in L_{\infty}(] 0, T[, \mathcal{L}(H))$ satisfying the inequality

$$
\left|\left(\left(d^{2} A^{-1}(t) / d t^{2}\right) g, v\right)\right| \leq c_{3}|g|\left(A^{-1}(t) v, v\right)^{1 / 2} \quad \forall g, v \in H .
$$

$A_{6}$. One has the inclusion $A_{1}(t)\left(d A^{-1}(t) / d t\right) \in B([0, T], \mathcal{L}(H))$ and the inequalities

$$
\begin{aligned}
\left|\left(A_{1}(t)\left(d A^{-1}(t) / d t\right) g, v\right)\right| & \leq c_{4}|g|\left(A^{-1}(t) v, v\right)^{1 / 2} \quad \forall g, v \in H, \\
-\operatorname{Re}\left(A_{1}(t) w, A(t) w\right) & \leq c_{5}(A(t) w, w) \quad \forall w \in D(A(t)) .
\end{aligned}
$$

The constants $c_{i} \geq 0, i=1, \ldots, 5$, in Conditions $A_{2}-A_{6}$ are assumed to be independent of $g, u$, $v, w$, and $t$.

The remaining conditions on the operators in Eq. (1) will be indicated in the statements of the theorems. Under these assumptions, we study the well-posed solvability of the nonlocal problem (1), (2) in the strong sense.

\section{CHOICE OF SPACES AND DEFINITION OF STRONG SOLUTIONS}

The nonlocal problem (1), (2) generates the linear unbounded operator $L \equiv\left\{\mathcal{L}(t), l_{0}, l_{1}\right\}$ : $E \supset D(L) \rightarrow F$ with dense domain

$$
D(L)=\left\{u \in \mathcal{H}: u \in D(A(t)), \frac{d u}{d t} \in D\left(A_{1}(t)\right), t \in[0, T] ; \frac{d u}{d t}, \frac{d^{2} u}{d t^{2}}, A(t) u, A_{1}(t) \frac{d u}{d t} \in \mathcal{H}\right\},
$$

where $\mathcal{H}=L_{2}(] 0, T[, H)$. Here the space of strong solutions of problem (1), (2) is the Banach space $E$ obtained as the closure of the set of all smooth solutions [functions in $D(L)$ ] in the norm

$$
\|u\|_{E}=\left(1-|\mu|^{2}\right)\left(1+|\mu|^{2}\right)^{-1}\left[\sup _{0<t<T}\left(|d u(u) / d t|^{2}+\left|A^{1 / 2}(t) u(t)\right|^{2}\right)\right]^{1 / 2} .
$$


The space of right-hand sides $\mathcal{F}=\{f(t), \varphi, \psi\}$ of problem (1), (2) is the Hilbert space $F=$ $\mathcal{H} \times W(0) \times H$ with finite Hermitian norm

$$
\|\mathcal{F}\|_{F}=\left[\|f\|_{0}^{2}+(4 T)^{-1}\left(\left|A^{1 / 2}(0) \varphi\right|^{2}+|\psi|^{2}\right)\right]^{1 / 2}, \quad\|f\|_{0}^{2}=\int_{0}^{T}|f(t)|^{2} d t,
$$

where the $W(t)$ are the Hilbert spaces obtained by equipping the domains $D\left(A^{1 / 2}(t)\right)$ of the operators $A^{1 / 2}(t)$ with the norms $|v|_{(t)}=\left|A^{1 / 2}(t) v\right|, t \in[0, T]$.

In a standard way, one can show that the operator $L$ admits a strong closure $\bar{L}$ in the product $E \times F$.

Lemma 1. If Condition $A_{1}$ is satisfied, the embedding $D(A(T)) \subset D(A(0))$ holds, and the set

$$
\tilde{D}(L)=\left\{v \in D(L): v \in D\left(A_{1}^{*}(t)\right), t \in[0, T] ; A_{1}^{*}(t) v \in \mathcal{H}\right\}
$$

is dense in $\mathcal{H}$, then the operator $L$ has a strong closure.

A function $u \in E$ belongs to the domain $D(\bar{L})$ of the closure $\bar{L}$ if there exists a sequence $u_{n} \in D(L)$ and an element $\mathcal{F} \in F$ such that $\left\|u_{n}-u\right\|_{E} \rightarrow 0$ and $\left\|L u_{n}-\mathcal{F}\right\|_{F} \rightarrow 0$ as $n \rightarrow \infty$. In this case, we set $\bar{L} u=\lim _{n \rightarrow \infty} L u_{n}=\mathcal{F}$.

Definition. The solutions $u \in D(\bar{L})$ of the operator equation $\bar{L} u=\mathcal{F}, \mathcal{F} \in F$, are called strong solutions of the nonlocal problem (1), (2).

\section{ENERGY INEQUALITY FOR STRONG SOLUTIONS}

For the strong solutions of the nonlocal problem (1), (2), we derive an energy inequality, which will imply the uniqueness and continuous dependence of these solutions on $f, \varphi$, and $\psi$.

Theorem 1. If the assumptions of Lemma 1 hold, Conditions $A_{2}-A_{4}$ are satisfied, and $|B(t) g| \leq c_{6}|g| \quad \forall g \in H, \quad\left|B_{0}(t) v\right| \leq c_{7}\left|A^{1 / 2}(t) v\right| \quad \forall v \in D\left(A^{1 / 2}(t)\right), \quad t \in[0, T], \quad c_{6}, c_{7} \geq 0$,

then the inequality

$$
\|u\|_{E}^{2} \leq 2 \gamma^{2} T(\gamma-4)^{-1}\|L u\|_{F}^{2} \quad \forall u \in D(\bar{L})
$$

holds for all $T<1 /\left(\gamma c_{8}\right)$ and $|\mu|^{2}<\left(1-\gamma c_{8} T\right) /\left(1+\gamma c_{8} T\right)$, where

$$
c_{8}=\min _{\varrho>0} \max \left\{2 c_{2}+2 c_{6}+\varrho^{-1} c_{7},\left(c_{1}+\varrho c_{7}\right) / 2\right\}
$$

and $\gamma>4$

Proof. We carry out the proof with the use of the smoothing operators $A_{\varepsilon}^{-1}(t)=(I+\varepsilon A(t))^{-1}$, $\varepsilon>0$, whose ranges coincide with $D(A(t))$. Their main properties are well known [2].

1. $\left|A_{\varepsilon}^{-1}(t) g-g\right| \rightarrow 0$ for all $g \in H$ uniformly with respect to $t$ as $\varepsilon \rightarrow 0$.

2. In the Hilbert space $H$, for all $t \in[0, T]$, there exists a strong derivative $d A_{\varepsilon}^{-1}(t) / d t \in$ $B([0, T], \mathcal{L}(H))$, and

$$
\frac{d\left(A(t) A_{\varepsilon}^{-1}(t)\right)}{d t}=-A(t) A_{\varepsilon}^{-1}(t) \frac{d A^{-1}(t)}{d t} A(t) A_{\varepsilon}^{-1}(t)
$$

By integrating by parts once with respect to $t$ from 0 to $\tau$ and by using the properties of the smoothing operators $A_{\varepsilon}^{-1}(t)$ and the estimate (3), for $c=0$, just as in [2], we obtain the inequality

$$
\left.(A(t) u, u)\right|_{t=\tau} \leq\left.(A(t) u, u)\right|_{t=0}+2 \operatorname{Re} \int_{0}^{\tau}\left(A(t) u, \frac{d u}{d t}\right) d t+c_{1} \int_{0}^{\tau}(A(t) u, u) d t
$$

for all $u \in D(L)$. 
By integrating by parts once with respect to $t$ from 0 to $\tau$, we obtain the identity

$$
\left.\left|\frac{d u}{d u}\right|^{2}\right|_{t=\tau}=2 \operatorname{Re} \int_{0}^{\tau}\left(\frac{d^{2} u}{d t^{2}}, \frac{d u}{d u}\right) d t+\left.\left|\frac{d u}{d u}\right|^{2}\right|_{t=0} \quad \forall u \in D(L) .
$$

We add inequality (10) and the identity (11), use the estimate (4), inequalities (8), and elementary estimates, and obtain the relation

$$
\begin{aligned}
\left.\left(\left|A^{1 / 2}(t) u\right|^{2}+\left|\frac{d u}{d t}\right|^{2}\right)\right|_{t=\tau} \leq 2 \int_{0}^{\tau}\left|\left(\mathcal{L}(t) u, \frac{d u}{d t}\right)\right| d t+\left(c_{1}+\varrho c_{7}\right) \int_{0}^{\tau}\left|A^{1 / 2}(t) u\right|^{2} d t \\
+\left(2 c_{2}+2 c_{6}+\varrho^{-1} c_{7}\right) \int_{0}^{\tau}\left|\frac{d u}{d t}\right|^{2} d t+\left.\left(\left|A^{1 / 2}(t) u\right|^{2}+\left|\frac{d u}{d t}\right|^{2}\right)\right|_{t=0} \quad \forall \varrho>0 .
\end{aligned}
$$

By a similar argument for the interval $[\tau, T]$ instead of $[0, \tau]$, for all $u \in D(L)$, we obtain the inequality

$$
\begin{gathered}
-\left.\frac{1+|\mu|^{2}}{2}\left(\left|A^{1 / 2}(t) u\right|^{2}+\left|\frac{d u}{d t}\right|^{2}\right)\right|_{t=\tau} \leq \frac{1+|\mu|^{2}}{2}\left[2 \int_{\tau}^{T}\left|\left(\mathcal{L}(t) u, \frac{d u}{d t}\right)\right| d t\right. \\
+\left(c_{1}+\varrho c_{7}\right) \int_{\tau}^{T}\left|A^{1 / 2}(t) u\right|^{2} d t+\left(2 c_{2}+2 c_{6}+\varrho^{-1} c_{7}\right) \int_{\tau}^{T}\left|\frac{d u}{d t}\right|^{2} d t \\
\left.-\left.\left(\left|A^{1 / 2}(t) u\right|^{2}+\left|\frac{d u}{d t}\right|^{2}\right)\right|_{t=T}\right]
\end{gathered}
$$

We add inequalities (12) and (13), minimize the right-hand side of the resulting inequality over all $\varrho>0$, and obtain the inequality

$$
\begin{aligned}
& \left.\frac{1-|\mu|^{2}}{2}\left(\left|A^{1 / 2}(t) u\right|^{2}+\left|\frac{d u}{d t}\right|^{2}\right)\right|_{t=\tau} \leq\left(1+|\mu|^{2}\right)\left[2 \int_{0}^{T}\left|\left(\mathcal{L}(t) u, \frac{d u}{d t}\right)\right| d t\right. \\
& \left.\quad+\min _{\varrho>0} \max \left\{2 c_{2}+2 c_{6}+\varrho^{-1} c_{7},\left(c_{1}+\varrho c_{7}\right) / 2\right\}\left(2 \int_{0}^{T}\left|A^{1 / 2}(t) u\right|^{2} d t+\int_{0}^{T}\left|\frac{d u}{d t}\right|^{2} d t\right)\right] \\
& \quad+\left.\left(\left|A^{1 / 2}(t) u\right|^{2}+\left|\frac{d u}{d t}\right|^{2}\right)\right|_{t=0}-\left.\frac{1+|\mu|^{2}}{2}\left(\left|A^{1 / 2}(t) u\right|^{2}+\left|\frac{d u}{d t}\right|^{2}\right)\right|_{t=T} \cdot
\end{aligned}
$$

Now we use the following lemma.

Lemma 2. Suppose that $g(0)-\mu g(T)=\varphi$ for some function $g \in C([0, T], H)$. Then the estimate

$$
|g(0)|^{2}-\frac{1+|\mu|^{2}}{2}|g(T)|^{2} \leq \frac{1+|\mu|^{2}}{1-|\mu|^{2}}|\varphi|^{2}
$$

holds for $|\mu|<1$. If the operator $A(t)$ satisfies Conditions $A_{1}$ and $A_{4}$ and one has the embedding $D(A(T)) \subset D(A(0))$, then the estimate

$$
\left|A^{1 / 2}(0) g(0)\right|^{2}-\frac{1+|\mu|^{2}}{2}\left|A^{1 / 2}(T) g(T)\right|^{2} \leq \frac{1+|\mu|^{2}}{1-|\mu|^{2}}\left|A^{1 / 2}(0) \varphi\right|^{2}
$$

holds for all $g \in D(A(T))$ and $\varphi \in D(A(0))$. 
Proof. The proof of the estimate (15) can be found in [1]. Let us prove the estimate (16). By virtue of Conditions $A_{1}$ and $A_{4}$ and the relation $-\mu g(T)=\varphi-g(0)$, we have

$$
\begin{aligned}
& -|\mu|^{2}\left|A^{1 / 2}(T) g(T)\right|^{2}=-|\mu|^{2}(A(T) g(T), g(T))=-(\tilde{A} \mu g(T), \mu g(T)) \\
& \quad=-(\tilde{A} \varphi-\tilde{A} g(0), \varphi-g(0))=-(\tilde{A} \varphi, \varphi)+(\tilde{A} \varphi, g(0))+(\tilde{A} g(0), \varphi)-(\tilde{A} g(0), g(0)) \\
& \quad=-(A(0) \varphi, \varphi)+(A(0) \varphi, g(0))+(A(0) g(0), \varphi)-(A(0) g(0), g(0)) \\
& \leq(\varepsilon-1)\left|A^{1 / 2}(0) g(0)\right|^{2}+\left(\varepsilon^{-1}-1\right)\left|A^{1 / 2}(0) \varphi\right|^{2} \quad \forall \varepsilon>0 .
\end{aligned}
$$

Here we set

$$
\varepsilon=\left(1-|\mu|^{2}\right)\left(1+|\mu|^{2}\right)^{-1}
$$

and obtain the estimate (16). The proof of Lemma 2 is complete.

On the right-hand side of inequality (14), we use the estimates (15) and (16) for $g=d u(t) / d t$ and $g=u$, respectively, divide both sides of the resulting inequality by $1+|\mu|^{2}$, make elementary estimates, and obtain the inequality

$$
\begin{gathered}
\left.\frac{1-|\mu|^{2}}{2\left(1+|\mu|^{2}\right)}\left(\left|A^{1 / 2}(t) u\right|^{2}+\left|\frac{d u}{d t}\right|^{2}\right)\right|_{t=\tau} \leq \frac{1}{\delta} \int_{0}^{T}|\mathcal{L}(t) u|^{2} d t+\delta \int_{0}^{T}\left|\frac{d u}{d t}\right|^{2} d t+2 c_{8} \int_{0}^{T}\left|A^{1 / 2}(t) u\right|^{2} d t \\
+c_{8} \int_{0}^{T}\left|\frac{d u}{d t}\right|^{2} d t+\frac{1}{1-|\mu|^{2}}\left(\left|A^{1 / 2}(0) l_{0} u\right|^{2}+\left|l_{1} u\right|^{2}\right) \quad \forall \delta>0 .
\end{gathered}
$$

This inequality, together with the estimate $c_{8} \leq \delta_{0}=\left(1-|\mu|^{2}\right)\left(\gamma T\left(1+|\mu|^{2}\right)\right)^{-1}$, which follows from the assumptions of Theorem 1, implies the inequality

$$
\left.\frac{\gamma T \delta_{0}}{2}\left(\left|A^{1 / 2}(t) u\right|^{2}+\left|\frac{d u}{d t}\right|^{2}\right)\right|_{t=\tau} \leq \delta_{0}^{-1}\|L u\|_{F}^{2}+2 \delta_{0}\left(\left\|A^{1 / 2}(t) u\right\|_{0}^{2}+\left\|\frac{d u}{d t}\right\|_{0}^{2}\right) .
$$

We integrate inequality (17) with respect to $\tau$ from 0 to $T$, divide by $T$, and obtain the estimate

$$
2 \delta_{0}\left(\left\|A^{1 / 2}(t) u\right\|_{0}^{2}+\left\|\frac{d u}{d t}\right\|_{0}^{2}\right) \leq 4\left((\gamma-4) \delta_{0}\right)^{-1}\|L u\|_{F}^{2}
$$

for all $\gamma>4$. In inequality (17), we take the least upper bound with respect to $\tau \in[0, T]$, use the estimate (18), and obtain

$$
T \delta_{0}\left(1+|\mu|^{2}\right)^{2}\left(1-|\mu|^{2}\right)^{-2}\|u\|_{E}^{2} \leq 2\left((\gamma-4) \delta_{0}\right)^{-1}\|L u\|_{F}^{2} \quad \forall u \in D(L) .
$$

Hence we have inequality (9) for smooth solutions $u \in D(L)$, and then we extend it by passing to the limit to all strong solutions $u \in D(\bar{L})$. The proof of Theorem 1 is complete.

Remark 1. If $c_{7}>0$ in inequality (8), then one can readily show that the constant $c_{8}$ attains the minimum value $c_{8}=\left(c_{1}+\varrho_{0} c_{7}\right) / 2$ for

$$
\varrho_{0}=\left(\sqrt{\left(c_{1}-4 c_{2}-4 c_{6}\right)^{2}+8 c_{7}^{2}}-c_{1}+4 c_{2}+4 c_{6}\right) /\left(2 c_{7}\right) .
$$

Corollary 1. The relation

$$
R(\bar{L})=\overline{R(L)}
$$

holds under the assumptions of Theorem 1 , where $R(\bar{L})$ is the range of the operator $\bar{L}$ and $\overline{R(L)}$ is the closure of the range $R(L)$ of the operator $L$ in $F$.

DIFFERENTIAL EQUATIONS $\quad$ Vol. $47 \quad$ No. $4 \quad 2011$ 


\section{THE RANGE IS DENSE}

Let us prove the following existence theorem for strong solutions of the nonlocal problem (1), (2).

Theorem 2. Let the assumptions of Theorem 1 hold, let Conditions $A_{5}$ and $A_{6}$ be satisfied, and let $\left(d A^{-1}(0) / d t\right) g=\left(d A^{-1}(T) / d t\right) g$ for all $g \in H$. Then, for any $T<1 /\left(\gamma c_{8}\right)$ and

$$
|\mu|^{2}<\left|\mu_{0}\right|^{2}=\min \left\{\left(\sqrt{c_{9}^{2} T^{2}+4}-c_{9} T\right) / 2,\left(1-\gamma c_{8} T\right) /\left(1+\gamma c_{8} T\right)\right\}
$$

where $\gamma$ and $c_{8}$ are defined in Theorem 1 and $c_{9}=3 c_{1}+2 c_{3}+c_{4}+c_{5}$, there exists a strong solution $u \in E$ of the nonlocal problem (1), (2) for any $f \in \mathcal{H}, \varphi \in W(0)$, and $\psi \in H$.

Proof. First, we carry out the proof for Eq. (1) with $B(t)=0$ and $B_{0}(t)=0$. By Corollary 1, it suffices to show that the set $R(L)$ is dense in $F$. Thus, assume that some nonzero element $V=\left\{v, \varphi_{1}, \psi_{1}\right\} \in F^{\prime}$ is orthogonal to $R(L)$; i.e.,

$$
\int_{0}^{T}(\mathcal{L}(t) u, v) d t+\frac{1}{4 T}\left(\left(A^{1 / 2}(0) l_{0} u, A^{1 / 2}(0) \varphi_{1}\right)+\left(l_{1} u, \psi_{1}\right)\right)=0 \quad \forall u \in D(L) .
$$

For $u \in D_{0}(L)=\left\{u \in D(L): l_{0} u=l_{1} u=0\right\}$, from identity (19), we obtain the identity

$$
\int_{0}^{T}(\mathcal{L}(t) u, v) d t=0 \quad \forall u \in D_{0}(L) .
$$

Lemma 3. Let the assumptions of Theorem 2 be satisfied. If identity (20) holds for some function $v \in \mathcal{H}$, then $v=0$.

Proof. We divide the proof into three stages.

1. For each parameter value $\tau, 0 \leq \tau<T$, in identity (20), one can set $u=A^{-1}(t) h$ for all $h \in M_{[\tau, T]}^{\mu}=\left\{h \in \mathcal{H}: d h / d t, d^{2} h / d t^{2} \in \mathcal{H}, h(t)=h(\tau), t \in[0, \tau[, h(\tau)-\mu h(T)=0\right.$, $d h(\tau) / d t-\mu d h(T) / d t=0\}$. Such functions $u$ satisfy homogeneous nonlocal conditions. Indeed, by virtue of the embedding $D(A(T)) \subset D(A(0))$, from Condition $A_{4}$, we have the relations $A(0) u=$ $\tilde{A} u=A(T) u$ and $u=A^{-1}(0) A(T) u$ for all $u \in D(A(T))$. The last relation permits one to find the first initial condition

$$
\begin{aligned}
l_{0} u & =A^{-1}(0) h(0)-\mu A^{-1}(T) h(T)=A^{-1}(0) A(T) A^{-1}(T) h(0)-\mu A^{-1}(T) h(T) \\
& =A^{-1}(T)[h(\tau)-\mu h(T)]=0 \quad \forall h \in M_{[\tau, T]}^{\mu} .
\end{aligned}
$$

By using the same relation $A^{-1}(0) A(T) u=u$ for all $u \in D(A(T))$ and the relation $\left(d A^{-1}(0) / d t\right) g=$ $\left(d A^{-1}(T) / d t\right) g$ for all $g \in H$, in a similar way from Theorem 2 , one can find the second initial condition

$$
\begin{aligned}
l_{1} u= & A^{-1}(0)(d h(0) / d t)-\mu A^{-1}(T)(d h(T) / d t)+\left(d A^{-1}(0) / d t\right) h(0)-\mu\left(d A^{-1}(T) / d t\right) h(T) \\
= & A^{-1}(T)[d h(\tau) / d t-\mu(d h(T) / d t)]+\left(d A^{-1}(0) / d t\right)[h(\tau)-\mu h(T)] \\
& +\mu\left(d A^{-1}(0) / d t\right) h(T)-\mu\left(d A^{-1}(T) / d t\right) h(T)=0 \quad \forall h \in M_{[\tau, T]}^{\mu} .
\end{aligned}
$$

Let $w \in \mathcal{H}$ be a solution of the nonlocal problem

$$
\left.\left.\frac{d w(t)}{d t}=v(t), \quad t \in\right] \tau, T\right], \quad w(\tau)-\mu w(T)=0
$$


and let $w(t)=w(\tau), t \in[0, \tau[$. As a result, from identity (20), we arrive at the identity

$$
\int_{\tau}^{T}\left(\frac{d^{2} h}{d t^{2}}, A^{-1}(t) \frac{d w}{d t}\right) d t=-\int_{\tau}^{T}\left(h, \frac{d w}{d t}\right) d t-\int_{\tau}^{T} \Phi(h, w) d t \quad \forall h \in M_{[\tau, T]}^{\mu},
$$

where

$$
\Phi(h, w)=\left(\frac{d^{2} A^{-1}(t)}{d t^{2}} h+2 \frac{d A^{-1}(t)}{d t} \frac{d h}{d t}+A_{1}(t) \frac{d A^{-1}(t)}{d t} h+A_{1}(t) A^{-1}(t) \frac{d h}{d t}, \frac{d w}{d t}\right) .
$$

The following assertion can be proved in a standard way.

Lemma 4. The adjoint operator of the operator $D=d / d t: \mathcal{H}_{\tau} \supset \mathcal{D}(D) \rightarrow \mathcal{H}_{\tau}$ with domain $\mathcal{D}(D)=\left\{\tilde{h} \in \mathcal{H}_{\tau}: d \tilde{h} / d t \in \mathcal{H}_{\tau}, \tilde{h}(\tau)-\mu \tilde{h}(T)=0\right\}$ is the operator $D^{*}=-d / d t$ with domain $\mathcal{D}\left(D^{*}\right)=\left\{\tilde{v} \in \mathcal{H}_{\tau}: d \tilde{v} / d t \in \mathcal{H}_{\tau}, \bar{\mu} \tilde{v}(\tau)-\tilde{v}(T)=0\right\}$.

Since, by virtue of the representation

$$
h(t)=\frac{1}{1-\mu}\left(\int_{\tau}^{t} h^{\prime}(s) d s+\mu \int_{t}^{T} h^{\prime}(s) d s\right)
$$

of functions $h \in M_{[\tau, T]}^{\mu}$, the right-hand side of identity (22) can be estimated from above by the norm of the derivative $d h / d t$ in $\mathcal{H}_{\tau}=L_{2}(] \tau, T[, H)$, it follows from Lemma 4 that the function $A^{-1}(t)(d w / d t)$ is differentiable with respect to $t$ in $\mathcal{H}_{\tau}$ and satisfies the adjoint nonlocal condition

$$
\bar{\mu} A^{-1}(\tau) \frac{d w(\tau)}{d t}-A^{-1}(T) \frac{d w(T)}{d t}=0 .
$$

Using this, we integrate by parts once on the left-hand side in identity (22) with respect to $t$, extend the result by passage to the limit to all $h \in \mathcal{H}_{\tau}$ such that $d h / d t \in \mathcal{H}_{\tau}$ and $h(\tau)-\mu h(T)=0$, set $h=w$, take the doubled real part, and obtain the relation

$$
-2 \operatorname{Re} \int_{\tau}^{T}\left(\frac{d w}{d t}, \frac{d}{d t}\left(A^{-1}(t) \frac{d w}{d t}\right)\right) d t=-2 \operatorname{Re} \int_{\tau}^{T}\left(w, \frac{d w}{d t}\right) d t-2 \operatorname{Re} \int_{\tau}^{T} \Phi(w, w) d t .
$$

Since the second derivative $d^{2} w / d t^{2}$ does not exist in $\mathcal{H}_{\tau}$, we cannot integrate by parts with respect to $t$ on the left-hand side in this relation; therefore, for simultaneously integrating by parts once with respect to $t$ and taking the adjoint of $A^{-1}(t)$, we need the following assertion.

Lemma 5 [2]. Let $X, Y$, and $Z$ be Banach spaces, let $S: X \rightarrow Y$ be a linear bounded operator, and let $P: Y \rightarrow Z$ be a linear closed operator with dense domain. If the domain of the product $P S$ is dense in $X$, then the adjoint operator $(P S)^{*}$ is equal to the weak closure of the product of the adjoint operators $S^{*}$ and $P^{*}$.

To apply Lemma 5 in the Hilbert spaces $X=Y=\mathcal{H}_{\tau}$ and $Z=\mathcal{H}_{\tau} \times H \times H$ to the linear bounded operator $S=A^{-1}(t): X \rightarrow Y$ and the linear closed operator $P g=\{d g / d t, g(\tau),-g(T)\}: Y \rightarrow Z$ with domain

$$
D(P)=\left\{g \in \mathcal{H}_{\tau}: d g / d t \in \mathcal{H}_{\tau}, \bar{\mu} g(\tau)-g(T)=0\right\},
$$

we find the adjoint operator of $P$.

Lemma 6. The adjoint operator of the operator $P g=\{d g / d t, g(\tau),-g(T)\}: \mathcal{H}_{\tau} \rightarrow \mathcal{H}_{\tau} \times H \times H$ with domain $D(P)=\left\{g \in \mathcal{H}_{\tau}: d g / d t \in \mathcal{H}_{\tau}, \bar{\mu} g(\tau)-g(T)=0\right\}$ is the operator $P^{*}(\{p(t), \tilde{\varphi}, \tilde{\psi}\})=$ $-d p / d t: \mathcal{H}_{\tau} \times H \times H \rightarrow \mathcal{H}_{\tau}$ with domain

$$
D^{*}=\left\{\{p(t), \tilde{\varphi}, \tilde{\psi}\} \in \mathcal{H}_{\tau} \times H \times H: d p / d t \in \mathcal{H}_{\tau}, p(\tau)-\mu p(T)=\tilde{\varphi}-\mu \tilde{\psi}\right\} .
$$

DIFFERENTIAL EQUATIONS $\quad$ Vol. $47 \quad$ No. $4 \quad 2011$ 
Proof. By the definition of the adjoint operator $P^{*}$, an element $\{p(t), \tilde{\varphi}, \tilde{\psi}\} \in \mathcal{H}_{\tau} \times H \times H$ belongs to the domain $D\left(P^{*}\right)$ if there exists a function $\tilde{w} \in \mathcal{H}_{\tau}$ such that

$$
\int_{\tau}^{T}\left(\frac{d g(t)}{d t}, p(t)\right) d t+(g(\tau), \tilde{\varphi})+(-g(T), \tilde{\psi})=\int_{\tau}^{T}(g(t), \tilde{w}) d t \quad \forall g \in D(P),
$$

and in this case, one has $\tilde{w} \equiv P^{*}(\{p(t), \tilde{\varphi}, \tilde{\psi}\})$.

The embedding $\mathcal{D}^{*} \subset D\left(P^{*}\right)$ is obvious. Let us prove the opposite embedding $D\left(P^{*}\right) \subset \mathcal{D}^{*}$. Let

$$
\{p(t), \tilde{\varphi}, \tilde{\psi}\} \in D\left(P^{*}\right) .
$$

It is well known that identity (25) implies that the generalized derivative $d p / d t \in \mathcal{H}_{\tau}$ exists for all $g \in D_{0}(P)=\{g \in D(P): g(\tau)=g(T)=0\}$. We integrate by parts once with respect to $t$ in identity $(25)$ for all $g \in D_{0}(P)$ and obtain $\tilde{w}=-d p / d t$ in $\mathcal{H}_{\tau}$; therefore, the identity acquires the form

$$
\int_{\tau}^{T}\left(\frac{d g(t)}{d t}, p(t)\right) d t+(g(\tau), \tilde{\varphi})+(-g(T), \tilde{\psi})=-\int_{\tau}^{T}\left(g(t), \frac{d p(t)}{d t}\right) d t \quad \forall g \in D(P) .
$$

Here we integrate by parts once on the right-hand side with respect to $t$, collect similar terms, take the function $g(t)=((1-\mu) t+\mu \tau-T)(\mu(p(T)-\tilde{\psi})+\tilde{\varphi}-p(\tau))$, which obviously belongs to $D(P)$, and obtain the relation $(\tau-T)|\mu(p(T)-\tilde{\psi})+\tilde{\varphi}-p(\tau)|^{2}=0$, which implies that $\tilde{\varphi}-\mu \tilde{\psi}=p(\tau)-\mu p(T)$. The proof of Lemma 6 is complete.

Note that the weak and strong closures of linear sets in Hilbert spaces coincide. The left-hand side of identity (22) can be represented in the form

$$
\int_{\tau}^{T}\left(\frac{d}{d t}\left(A^{-1}(t) \frac{d h}{d t}\right), \frac{d w}{d w}\right) d t-\int_{\tau}^{T}\left(\frac{d A^{-1}(t)}{d t} \frac{d h}{d t}, \frac{d w}{d t}\right) d t,
$$

where the second integral and, as is well-known, the right-hand side of this identity can be estimated from above by the norm of the function $d h / d t$ in $\mathcal{H}_{\tau}$. It follows that the function $d w / d t$ belongs to the domain $D\left((P S)^{*}\right)$ of the adjoint operator $(P S)^{*}$; i.e., by Lemma $5,(P S)^{*}=\overline{S^{*} P^{*}}$, and there exists a sequence $p_{n}(t)$ such that $d p_{n} / d t \in D\left(P^{*}\right)$ and $d p_{n} / d t \rightarrow d w / d t$ in $\mathcal{H}_{\tau}$ as $n \rightarrow \infty$.

By applying Lemma 6 to the first three terms of the expressions

$$
\begin{aligned}
J_{n}= & -\int_{\tau}^{T}\left(\frac{d p_{n}}{d t}, \frac{d}{d t}\left(A^{-1}(t) \frac{d w}{d t}\right)\right) d t-\left(\tilde{\varphi}_{n}, A^{-1}(\tau) \frac{d w(\tau)}{d t}\right)-\left(\tilde{\psi}_{n},-A^{-1}(T) \frac{d w(T)}{d t}\right) \\
& +\left(\tilde{\varphi}_{n}, A^{-1}(\tau) \frac{d w(\tau)}{d t}\right)+\left(\tilde{\psi}_{n},-A^{-1}(T) \frac{d w(T)}{d t}\right), \quad\left\{\frac{d p_{n}}{d t}, \tilde{\varphi}_{n}, \tilde{\psi}_{n}\right\} \in D\left(P^{*}\right),
\end{aligned}
$$

for all $\tau \in[0, T[$, we obtain the expressions

$$
J_{n}=\int_{\tau}^{T}\left(A^{-1}(t) \frac{d^{2} p_{n}}{d t^{2}}, \frac{d w}{d t}\right) d t+\left(\tilde{\varphi}_{n}, A^{-1}(\tau) \frac{d w(\tau)}{d t}\right)+\left(\tilde{\psi}_{n},-A^{-1}(T) \frac{d w(T)}{d t}\right) .
$$

By virtue of the nonlocal condition (23), the condition

$$
\tilde{\varphi}_{n}-\mu \tilde{\psi}_{n}=\left(d p_{n}(\tau) / d t\right)-\mu\left(d p_{n}(T) / d t\right),
$$


and Lemma 6, the sum of the last two terms in the expressions (27) is equal to

$$
\left(\left(d p_{n}(\tau) / d t\right)-\mu\left(d p_{n}(T) / d t\right), A^{-1}(\tau)(d w(\tau) / d t)\right)
$$

for all $\tau \in\left[0, T\left[\right.\right.$. By Lemma 5 , the value of the adjoint operator $(P S)^{*}$ on $d w / d t$ in $\mathcal{H}_{\tau}$ is equal to

$$
\begin{aligned}
(P S)^{*} \frac{d w}{d t} & =\overline{S^{*} P^{*}} \frac{d w}{d t}=-\lim _{n \rightarrow \infty} A^{-1}(t) \frac{d^{2} p_{n}}{d t^{2}}=\lim _{n \rightarrow \infty}\left(-\frac{d}{d t} A^{-1}(t)+\frac{d A^{-1}(t)}{d t}\right) \frac{d p_{n}}{d t} \\
& =\lim _{n \rightarrow \infty}\left(-\frac{d}{d t} A^{-1}(t) \frac{d p_{n}}{d t}+\frac{d A^{-1}(t)}{d t} \frac{d p_{n}}{d t}\right)=-\frac{d}{d t}\left(A^{-1}(t) \frac{d w}{d t}\right)+\frac{d A^{-1}(t)}{d t} \frac{d w}{d t} .
\end{aligned}
$$

Using this, in the expressions (26) and (27), we pass to the limit as $n \rightarrow \infty$, take the doubled real part, and obtain the value of the left-hand side of relation (24) for almost all $\tau \in[0, T[:$

$$
-\int_{\tau}^{T}\left(\frac{d A^{-1}(t)}{d t} \frac{d w}{d t}, \frac{d w}{d t}\right) d t+\left(\frac{d w(\tau)}{d t}-\mu \frac{d w(T)}{d t}, A^{-1}(\tau) \frac{d w(\tau)}{d t}\right) .
$$

By integrating once by parts with respect to $t$ and by taking into account the initial condition in (21), we obtain

$$
-2 \operatorname{Re} \int_{\tau}^{T}\left(w, \frac{d w}{d t}\right) d t=-\left(|\mu|^{-2}-1\right)|w(\tau)|^{2}
$$

for all $\tau \in[0, T[$.

From relations (24) and (29) and from the expression (28), we obtain

$$
\left(\frac{d w(\tau)}{d t}-\mu \frac{d w(T)}{d w}, A^{-1}(\tau) \frac{d w(\tau)}{d t}\right)+\left(|\mu|^{-2}-1\right)|w(\tau)|^{2}=\int_{\tau}^{T} \Phi_{1}(w, w) d t
$$

for almost all $\tau \in\left[0, T\left[\right.\right.$, where $\Phi_{1}(w, w)=-2 \operatorname{Re} \Phi(w, w)+\left(\left(d A^{-1}(t) / d t\right)(d w / d t), d w / d t\right)$.

By Condition $A_{4}$ and (23), we have

$$
\begin{aligned}
d w(\tau) / d t-\mu d w(T) / d t & =\tilde{A}\left(A^{-1}(\tau)(d w(\tau) / d t)-\mu A^{-1}(T)(d w(T) / d t)\right) \\
& =\left(1-|\mu|^{2}\right) \tilde{A} A^{-1}(\tau)(d w(\tau) / d t)=\left(1-|\mu|^{2}\right)(d w(\tau) / d t) .
\end{aligned}
$$

It follows that relation (30) acquires the form

$$
\left(1-|\mu|^{2}\right)\left|A^{-1 / 2}(\tau) \frac{d w(\tau)}{d t}\right|^{2}+\left(|\mu|^{-2}-1\right)|w(\tau)|^{2}=\int_{\tau}^{T} \Phi_{1}(w, w) d t
$$

for almost all $\tau \in[0, T[$.

2. For each $\tau, 0<\tau \leq T$, in identity (20), one can set $u=A^{-1}(t) h$ for all

$$
\begin{gathered}
\left.h \in M_{[\tau, T]}^{\mu}=\left\{h \in \mathcal{H}: d h / d t, d^{2} h / d t^{2} \in \mathcal{H}, h(t)=h(\tau), t \in\right] \tau, T\right], \\
h(0)-\mu h(\tau)=0, d h(0) / d t-\mu d h(\tau) / d t=0\} .
\end{gathered}
$$

By analogy with the first stage, one can show that $u \in D_{0}(L)$.

Let $w \in \mathcal{H}$ be a solution of the nonlocal problem

$$
\left.\frac{d w(t)}{d t}=v(t), \quad t \in\right] 0, \tau[, \quad w(0)-\mu w(\tau)=0
$$

DIFFERENTIAL EQUATIONS $\quad$ Vol. $47 \quad$ No. 42011 
and let $w(t)=w(\tau)$ for all $t \in] \tau, T]$. In the argument of the first stage, we set $\tau=0$ and $T=\tau$ and obtain the relation

$$
\left(|\mu|^{-2}-1\right)\left|A^{-1 / 2}(\tau) \frac{d w(\tau)}{d t}\right|^{2}+\left(1-|\mu|^{2}\right)|w(\tau)|^{2}=\int_{0}^{\tau} \Phi_{1}(w, w) d t
$$

for almost all $\tau \in] 0, T]$.

3. By adding relations (31) and (32) term by term, for almost all $\tau \in] 0, T[$, we obtain the relation

$$
\left(|\mu|^{-2}-|\mu|^{2}\right)\left(\left|A^{-1 / 2}(\tau) \frac{d w(\tau)}{d t}\right|^{2}+|w(\tau)|^{2}\right)=\int_{0}^{T} \Phi_{1}(w, w) d t .
$$

By virtue of inequalities (3), (5), (6), and (7), we have the upper bound

$$
\Phi_{1}(w, w) \leq\left(3 c_{1}+2 c_{3}+c_{4}+c_{5}\right)\left(\left|A^{-1 / 2}(t) \frac{d w}{d t}\right|^{2}+|w|^{2}\right) .
$$

In (33), we use this estimate, integrate the resulting relation with respect to $\tau$ from 0 to $T$, and obtain the inequality

$$
\left(|\mu|^{-2}-|\mu|^{2}-\left(3 c_{1}+2 c_{3}+c_{4}+c_{5}\right) T\right) \int_{0}^{T}\left(\left|A^{-1 / 2}(t) \frac{d w}{d t}\right|^{2}+|w|^{2}\right) d t \leq 0 .
$$

Hence it follows that $d w / d t=0$ for all $\mu$ and $T$ described in Theorem 2, and hence $v=0$ in $\mathcal{H}$. The proof of Lemma 3 is complete.

By Lemma 3, it follows from identity (19) that

$$
\left(A^{1 / 2}(0) l_{0} u, A^{1 / 2}(0) \varphi_{1}\right)+\left(l_{1} u, \psi_{1}\right)=0 \quad \forall u \in D(L) .
$$

In this identity, we set $u=t(T-t)^{2} A^{-1}(t) h$ for all $h \in H$ and obtain $\psi_{1}=0$. Then in identity (34) we set $u=(T-t) A^{-1}(t) h$ for all $h \in H$ and obtain $\varphi_{1}=0$. Therefore, $V=\left\{v, \varphi_{1}, \psi_{1}\right\}=0$, which contradicts the original assumption that $V \neq 0$.

The proof of Theorem 2 for the case in which $B(t) \neq 0$ and $B_{0}(t) \neq 0$ can be performed by the standard method of continuation with respect to a parameter. The proof of Theorem 2 is complete.

Remark 2. The nonlocal problem (1), (2) with nonlocality parameter $|\mu|>1$ can be reduced by the change of variables $t^{\prime}=T-t$ to the above-considered nonlocal problem (1), (2).

Remark 3. If the operator $A(t)=A$ is independent of $t$ and $A_{1}(t)=B(t)=B_{0}(t)=0$ in Eq. (1), then in Theorems 1 and 2, one can take $t \in[0, T]$ for all $T<+\infty$ and $|\mu|<1(|\mu|>1$ by Remark 2), because $c_{i}=0, i=1, \ldots, 8$. In this case, problem (1), (2) and the assertions of Theorems 1 and 2 coincide with the problem and the assertions of Theorems 1 and 2 in [1] for $\lambda=0$.

\section{MIXED PROBLEM FOR THE STRING VIBRATION EQUATION WITH TIME-DEPENDENT BOUNDARY CONDITIONS AND NONLOCAL INITIAL CONDITIONS}

In the domain $G=] 0, l[\times] 0, T[$ of independent variables $x$ and $t$, consider the complete equation

$$
\mathcal{L}(t) u \equiv u_{t t}+a_{1}(x, t) u_{x t}-\left(a(x) u_{x}\right)_{x}+b_{2}(x, t) u_{t}+b_{1}(x, t) u_{x}+b_{0}(x, t) u=f(x, t)
$$

of forced vibrations of a string with the $t$-dependent boundary conditions

$$
\left.\left[u_{x}-\alpha(t) u\right]\right|_{x=0}=0,\left.\quad\left[u_{x}+\beta(t) u\right]\right|_{x=l}=0, \quad t \in[0, T],
$$

and the time-nonlocal conditions

$$
l_{0} u \equiv u(x, 0)-\mu u(x, T)=\varphi(x), \quad l_{1} u \equiv u_{t}(x, 0)-\mu u_{t}(x, T)=\psi(x), \quad|\mu|<1 .
$$


Theorem 3. Let the coefficients of Eq. (35) satisfy the conditions $a \in C^{(1)}[0, l]$ and $a(x) \geq$ $a_{0}>0$ for $x \in[0, l], a_{1},\left(a_{1}\right)_{x} \in C(\bar{G}), a_{1}(0, t) \leq 0$, and $a_{1}(l, t) \geq 0$ for $t \in[0, T]$, and $b_{i} \in C(\bar{G})$ for $i=0,1,2$, and let the coefficients of the boundary conditions satisfy the conditions $\alpha, \beta \in C^{(2)}[0, T]$, $\alpha(t), \beta(t) \geq 0$, and $\alpha(t)+\beta(t)>0$ for $t \in[0, T], \alpha(0)=\alpha(T), \beta(0)=\beta(T), \alpha^{\prime}(0)=\alpha^{\prime}(T)$, and $\beta^{\prime}(0)=\beta^{\prime}(T)$. Then the mixed problem (35)-(37) is well posed in the strong sense for the admissible intervals $] 0, T[$ and $]-\mu_{0}, \mu_{0}$ [ defined in Theorem 2 , for the constants $c_{i}, i=1, \ldots, 8$, computed below, and for arbitrary $f(x, t) \in L_{2}(G), \varphi(x) \in \tilde{W}_{2,0}^{1}(0, l)$ (this Hilbert space is defined in the proof $)$, and $\psi(x) \in L_{2}(0, l)$.

Proof. The mixed problem (35)-(37) is a special case of the abstract nonlocal problem (1), (2). For it, let us verify the assumptions of Theorems 1 and 2 in the Hilbert space $H=L_{2}(0, l)$ with norm $\|\cdot\|$ and inner product $(\cdot, \cdot)$. For each $t \in[0, T]$, the linear operators $A(t) u=-\left(a(x) u_{x}\right)_{x}$ are obtained as the restrictions to their domains $D(A(t))=\left\{u \in W_{2}^{2}(0, l): u \in(36)\right\}, t \in[0, T]$, of the bounded linear operator $\tilde{A}=-\left(a(x) u_{x}\right)_{x}$ acting from the Sobolev space $V=W_{2}^{2}(0, l)$ to $L_{2}(0, l)$.

They are obviously symmetric, positive, and self-adjoint in $L_{2}(0, l)$, since on the entire $L_{2}(0, l)$, they have bounded inverses

$$
\begin{aligned}
A^{-1}(t) g & =-J(x) g+p_{1}(t) \int_{0}^{l} g(s) d s+p_{2}(t) J(l) g+\left(p_{3}(t) \int_{0}^{l} g(s) d s+p_{4}(t) J(l) g\right) \int_{0}^{x} \frac{d s}{a(s)}, \\
J(x) g & =\int_{0}^{x} \frac{1}{a(s)} \int_{0}^{s} g(\tau) d \tau d s, \quad p_{1}(t)=\left(a(0) \alpha(t)+a(0) a(l) \alpha(t) \beta(t) \int_{0}^{l} \frac{d s}{a(s)}+a(l) \beta(t)\right)^{-1}, \\
p_{2}(t) & =a(l) \beta(t) p_{1}(t), \quad p_{3}(t)=a(0) \alpha(t) p_{1}(t), \quad p_{4}(t)=a(0) a(l) \alpha(t) \beta(t) p_{1}(t) .
\end{aligned}
$$

Their boundedness follows from the estimate $\left\|A^{-1}(t) g\right\| \leq c_{10}\|g\|$ for all $g \in L_{2}(0, l)$, where

$$
c_{10}=l \max _{t \in[0, T]}\left(\left|p_{1}(t)\right|+\int_{0}^{l} \frac{d s}{a(s)}\left(1+\left|p_{2}(t)\right|+\left|p_{3}(t)\right|+\left|p_{4}(t)\right| \int_{0}^{l} \frac{d s}{a(s)}\right)\right) .
$$

By $\dot{r}(t)$ and $\ddot{r}(t)$, we denote the first and second derivatives of a numerical function $r(t)$. For $\alpha(t), \beta(t) \in C^{(1)}[0, T]$, in $L_{2}(0, l)$, there exists a strong derivative

$$
\frac{d A^{-1}(t)}{d t} g=\dot{p}_{1}(t) \int_{0}^{l} g(s) d s+\dot{p}_{2}(t) J(l) g+\left(\dot{p}_{3}(t) \int_{0}^{l} g(s) d s+\dot{p}_{4}(t) J(l) g\right) \int_{0}^{x} \frac{d s}{a(s)} .
$$

It is bounded by virtue of the estimate $\left\|\left(d A^{-1}(t) / d t\right) g\right\| \leq c_{11}\|g\|$ for all $g \in L_{2}(0, l)$ with

$$
c_{11}=4 l \max _{t \in[0, T]}\left\{\left|\dot{p}_{1}(t)\right|,\left|\dot{p}_{2}(t)\right| \int_{0}^{l} \frac{d s}{a(s)},\left|\dot{p}_{3}(t)\right| \int_{0}^{l} \frac{d s}{a(s)},\left|\dot{p}_{4}(t)\right|\left(\int_{0}^{l} \frac{d s}{a(s)}\right)^{2}\right\} .
$$

In inequality (3), we set $g=A(t) v$ and $v \in D(A(t))$ and derive the equivalent inequality

$$
\begin{aligned}
& -\left(\frac{d A^{-1}(t)}{d t} A(t) v, A(t) v\right) \leq \frac{c_{11}}{4 l}\left(\left.a(x) v_{x}\right|_{0} ^{l}+\left(\int_{0}^{l} a(x)\left|v_{x}\right|^{2} d x\right)^{1 / 2}\right)^{2} \leq c_{1}\left\|A^{1 / 2}(t) v\right\|^{2}, \\
& \left\|A^{1 / 2}(t) v\right\|=\sqrt{\frac{a(0)\left(\alpha(t)|v(0)|^{2}+\left|v_{x}(0)\right|^{2}\right)}{1+\alpha(t)}+\frac{a(l)\left(\beta(t)|v(l)|^{2}+\left|v_{x}(l)\right|^{2}\right)}{1+\beta(t)}+\int_{0}^{l} a(x)\left|v_{x}\right|^{2} d x,} \\
& c_{1}=\left(c_{11} /(4 l)\right) \max _{t \in[0, T]}(\sqrt{a(0)(1+\alpha(t))}+\sqrt{a(l)(1+\beta(t))}+1)^{2} .
\end{aligned}
$$


The Hilbert spaces $\tilde{W}_{2, t}^{1}(0, l)$ are the closures of the sets $\left\{v \in W_{2}^{2}(0, l): v \in(36)\right\}$ in the Hermitian norms $(39), t \in[0, T]$.

For arbitrary $t \in[0, T]$, the linear operator $A_{1}(t) u=a_{1}(x, t) u_{x}$ with domain $D\left(A_{1}(t)\right)=$ $W_{2}^{1}(0, l)$ satisfies the estimate $\left\|A_{1}(t) v\right\| \leq c_{12}\left\|A^{1 / 2}(t) v\right\|$ for all $v \in D(A(t))$ with constant $c_{12}=$ $a_{0}^{-1 / 2} \max _{\{x, t\} \in G}\left|a_{1}(x, t)\right|$; i.e., $A_{1}(t) A^{-1 / 2}(t) \in B\left([0, T], L_{2}(0, l)\right)$. By integrating by parts once with respect to $x$ and by taking into account the inequalities $a_{1}(l, t) \geq 0$ and $a_{1}(0, t) \leq 0$, we obtain inequality (4),

$$
-\operatorname{Re}\left(A_{1}(t) u, u\right)=-\left.\frac{a_{1}(x, t)}{2}|u|^{2}\right|_{0} ^{l}+\frac{1}{2} \int_{0}^{l}\left(a_{1}(x, t)\right)_{x}|u|^{2} d x \leq c_{2}\|u\|^{2} \quad \forall u \in D(A(t)),
$$

where $c_{2}=(1 / 2) \max _{\{x, t\} \in \bar{G}}\left|\left(a_{1}(x, t)\right)_{x}\right|$.

Without loss of generality, in inequality (6), we take $v=A(t) u, u \in D(A(t))$, and obtain

$$
\begin{aligned}
& \left|\left(A_{1}(t) \frac{d A^{-1}(t)}{d t} g, A(t) u\right)\right|=\left|\int_{0}^{l} \frac{a_{1}(x, t)}{a(x)}\left(\dot{p}_{3}(t) \int_{0}^{l} g(x) d x+\dot{p}_{4}(t) J(l) g\right)\left(-a(x) u_{x}\right)_{x} d x\right| \\
& \leq\left|\dot{p}_{3}(t)+\dot{p}_{4}(t) \int_{0}^{l} \frac{d x}{a(x)}\right| \sqrt{l}\|g\| \max _{x \in[0, l]}\left|\frac{a_{1}(x, t)}{a(x)}\right|\left|a(x) u_{x}\right|_{0}^{l} \mid \leq c_{4}\|g\|\left\|A^{1 / 2}(t) u\right\|, \\
& c_{4}=\sqrt{l} \max _{\{x, t\} \in \bar{G}}\left(\left|\dot{p}_{3}(t)+\dot{p}_{4}(t) \int_{0}^{l} \frac{d x}{a(x)}\right|\left|\frac{a_{1}(x, t)}{a(x)}\right|(\sqrt{a(0)(1+\alpha(t))}+\sqrt{a(l)(1+\beta(t))})\right) .
\end{aligned}
$$

Hence one can readily find that the operators $A_{1}(t)\left(d A^{-1}(t) / d t\right) \in B\left([0, T], L_{2}(0, l)\right)$ are bounded. By integrating by parts once with respect to $x$, we obtain

$$
\begin{gathered}
-\operatorname{Re}\left(A_{1}(t) w, A(t) w\right)=\left.\frac{a_{1}(x, t) a(x)}{2}\left|w_{x}\right|^{2}\right|_{0} ^{l}+\frac{1}{2} \int_{0}^{l} \frac{\partial}{\partial x}\left(\frac{a_{1}(x, t)}{a(x)}\right) a^{2}(x)\left|w_{x}\right|^{2} d x \leq c_{5}\left\|A^{1 / 2}(t) w\right\|^{2}, \\
c_{5}=\frac{1}{2} \max _{t \in[0, T]}\left\{a_{1}(l, t)(1+\beta(t)),-a_{1}(0, t)(1+\alpha(t)), \max _{x \in[0, l]}\left|\left(\frac{a_{1}(x, t)}{a(x)}\right)_{x} a(x)\right|\right\} .
\end{gathered}
$$

If $\alpha(t), \beta(t) \in C^{(2)}[0, T]$, then in $L_{2}(0, l)$, there exists a strong second derivative

$$
\frac{d^{2} A^{-1}(t)}{d t^{2}} g=\ddot{p}_{1}(t) \int_{0}^{l} g(s) d s+\ddot{p}_{2}(t) J(l) g+\left(\ddot{p}_{3}(t) \int_{0}^{l} g(s) d s+\ddot{p}_{4}(t) J(l) g\right) \int_{0}^{x} \frac{d s}{a(s)} .
$$

In inequality (5), we set $v=A(t) u$ and obtain the equivalent inequality

$$
\begin{aligned}
& \left|\left(\frac{d^{2} A^{-1}(t)}{d t^{2}} g, A(t) u\right)\right| \leq c_{13} \int_{0}^{l}|g(x)| d x\left|a(x) u_{x}\right|_{0}^{l} \mid \leq c_{3}\|g\|\left\|A^{1 / 2}(t) u\right\|, \quad u \in D(A(t)), \\
& c_{13}=\max _{t \in[0, T]}\left\{\left|\ddot{p}_{1}(t)\right|+\left|\ddot{p}_{2}(t)\right| \int_{0}^{l} \frac{d s}{a(s)},\left|\ddot{p}_{3}(t)\right| \int_{0}^{l} \frac{d s}{a(s)}+\left|\ddot{p}_{4}(t)\right|\left(\int_{0}^{l} \frac{d s}{a(s)}\right)^{2}\right\}, \\
& c_{3}=\sqrt{l} c_{13} \max _{t \in[0, T]}(\sqrt{a(l)(1+\beta(t))}+\sqrt{a(0)(1+\alpha(t))}) .
\end{aligned}
$$

This inequality implies that the operators $d^{2} A^{-1}(t) / d t^{2} \in L_{\infty}(] 0, T\left[, L_{2}(0, l)\right)$ are bounded. 
The operators $B(t) u=b_{2}(x, t) u$ and $B_{0}(t) u=b_{1}(x, t) u_{x}+b_{0}(x, t) u$ with domains $D(B(t))=$ $L_{2}(0, l)$ and $D\left(B_{0}(t)\right)=W_{2}^{1}(0, l)$, respectively, satisfy inequalities (8) with constants

$$
c_{6}=\max _{\{x, t\} \in \bar{G}}\left|b_{2}(x, t)\right|, \quad c_{7}=\max _{\{x, t\} \in \bar{G}}\left(a_{0}^{-1 / 2}\left|b_{1}(x, t)\right|+\sqrt{c_{10}}\left|b_{0}(x, t)\right|\right) .
$$

For functions $\alpha(t), \beta(t) \in C^{(1)}[0, T]$ such that $\alpha(0)=\alpha(T), \beta(0)=\beta(T), \alpha^{\prime}(0)=\alpha^{\prime}(T)$, and $\beta^{\prime}(0)=\beta^{\prime}(T)$, from $(38)$, we have $\left(d A^{-1}(0) / d t\right) g=\left(d A^{-1}(T) / d t\right) g$ for all $g \in L_{2}(0, l)$.

The set $\tilde{D}(L)$ for problem (35)-(37) is obviously dense in $\mathcal{H}=L_{2}(G)$, because it contains the set $C_{0}^{\infty}(G)$ of all infinitely differentiable functions compactly supported in $G$, which is dense in $\mathcal{H}$. The proof of Theorem 3 is complete.

Remark 4. In Theorem 3, the requirement $\alpha(t)+\beta(t)>0$ is only imposed to simplify the verification of the assumptions of Theorems 1 and 2 . One can readily justify Theorem 3 without this requirement if, when verifying Theorems 1 and 2 , one replaces the operators $A(t)$ and $B_{0}(t)$ by the corresponding operators $A(t)+\varepsilon_{0}$ and $B_{0}(t)-\varepsilon_{0}, \varepsilon_{0}>0$.

\section{ACKNOWLEDGMENTS}

The research was supported by the Belarus Republic Foundation for Basic Research (project no. F07K-016).

\section{REFERENCES}

1. Chesalin, V.I. and Yurchuk, N.I., Problem with Nonlocal Conditions for Abstract Love Equations, Vestsi Akad Navuk BSSR Ser. Fiz.-Mat. Navuk, 1973, no. 6, pp. 30-35.

2. Lomovtsev, F.E., Necessary and Sufficient Conditions for the Unique Solvability of the Cauchy Problem for Second-Order Hyperbolic Differential Equations with Variable Domain of Operator Coefficients, Differ. Uravn., 1992, vol. 28, no. 5, pp. 873-886.

3. Lomovtsev, F.E., Second-Order Hyperbolic Operator-Differential Equations with Variable Domains of Smooth Operator Coefficients, Dokl. Nats. Akad. Navuk Belarusi, 2001, vol. 45, no. 1, pp. 34-37.

4. Krein, S.G., Lineinye differentsial'nye uravneniya $v$ banakhovykh prostranstvakh (Linear Differential Equations in a Banach Space), Moscow: Nauka, 1967. 